\title{
Audiometric Hearing Results After Ossicular Chain Reconstruction with Partial Titanium Clip Prostheses
}

\author{
Nayellin Reyes Chicuellar ${ }^{1 *}$, Anthony Noor ${ }^{1}$ and Thomas Kertesz ${ }^{2}$ \\ ${ }^{1}$ Department of Otolaryngology, Royal Darwin Hospital, Australia
}

${ }^{2}$ Department of Otolaryngology, Prince of Wales Hospital, Australia

*Corresponding author: Nayellin Reyes Chicuellar, Department of Otolaryngology, Royal Darwin Hospital, Northern Territory, Australia

\begin{abstract}
Objective: This paper reviews the outcomes of partial ossicular chain reconstruction using the Kurz Clip-Piston Dresden prosthesis (Clip) in comparison to the earlier generation Bell prosthesis (Bell).

Study design: A retrospective monocentric chart review.

Subjects and Methods: All patients undergoing ossicular chain reconstruction between 1 January 2014 and 31 December 2018.

Results: Forty-three patients, 7 children and 35 adults were included in the study. A successful hearing result was defined as $\mathrm{ABG}<20 \mathrm{~dB}$ [1]. A total of $91 \%$ of cases resulted in an overall successful hearing. The overall PTA improved by $20 \mathrm{~dB}+/-0.63$ (preop: $52.6+/-19$, postop: $32.6+/-14.8$ ). The overall ABG improvement was $33.9+/-11.4$ (preop: $13+/-7.6$, postop: $20.7+/-3.8$ ). The overall results of Clip being better (96\%) than the results for Bell prosthesis (82\%). A successful postoperative ABG of $<20 \mathrm{~dB}$ was obtained in $91 \%$ of the patients $(n=39)$. The Clip prosthesis had a significantly higher success rate of $96 \%$ ( $n=26)$ in comparison to the Bell prosthesis which was successful in $82 \%$ of patients $(n=14)(p<0.001)$. Results showed a low complication rate. One revision procedure (2.3\%) to modify the length was needed with a Clip prosthesis and one revision procedure was required in the Bell group due to extrusion; it was replaced with a Clip prosthesis
\end{abstract}

Conclusion: Clip prosthesis demonstrates promising outcomes partial ossicular chain reconstruction with higher rates of postoperative air-bone Gap reduction and minimal complications.

Keywords: Titanium Clip-Piston Dresden Prosthesis; Old-style Bell Partial Prosthesis; Ossicular Chain Reconstruction; Air-Bone Gap

Abbreviations: Clip: Kurz Clip-Piston Dresden Prosthesis; Bell: Bell prosthesis; PORP: Partial Ossicular Replacement Prosthesis; CSOM: Chronic Suppurative Otitis Media

\section{Introduction}

Ossicular chain reconstruction remains the primary method of restoring conductive hearing deficits commonly seen in chronic middle ear disease [2,3]. Since 1994, the German company Kurz has developed different forms of titanium ossicular prostheses. The earlier style Bell prosthesis (Bell) was designed as a partial ossicular replacement prosthesis (PORP) to be placed on the intact stapes capitulum (Figure 1). Design developments led to the newer

Clip-Piston Dresden Prosthesis (Clip) which features a springloaded fastening mechanism to secure the coupling to a mobile stapes (Figure 2). The design is reported to reduce dislocation and extrusion of prosthesis with superior audiological outcomes. The purpose of this study is to compare postoperative audiological outcomes and complications of the Clip prosthesis with the Bell prosthesis. 


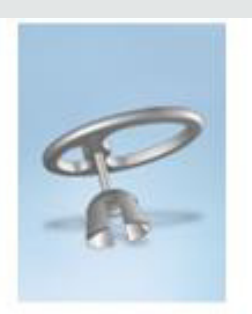

Earlier Style Bell Partial Prosthesis Images reproduction with the authorization of Heinz Kurz $\mathrm{GmbH}$ Medizinetechnik.

Figure 1.

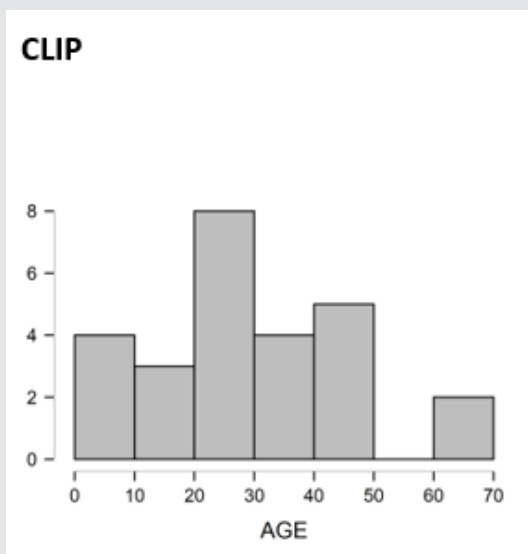

\section{BELL}

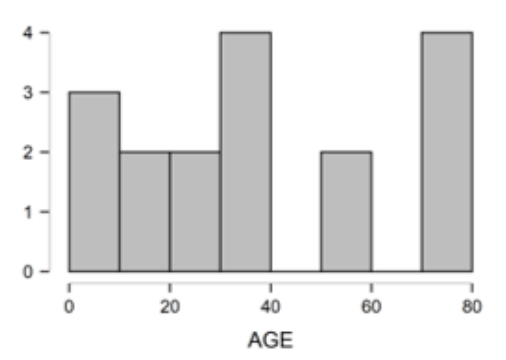

Figure 2: Age histogram.

\section{Materials and Methods}

Following institutional review board approval (no. 004834UNSW), A de-personalized chart review of all patients who underwent partial ossicular replacement using a Kurz titanium prosthesis from January 2014 to December 2018 was undertaken. All operation was performed by a single surgeon, the senior author (TRK). All the patients had cartilage interposition grafts between the prosthesis and the tympanic membrane. Cohorts were stratified based on the use of either the Kurz Clip-Piston Dresden or the oldstyle Bell Prosthesis. All procedures were analyzed in a postsurgical data base. Pre-operative and post-operative audiograms recorded thresholds at 0.5, 1, 2 and $3 \mathrm{KHz}$ according to AAO-HNS guidelines. All patients completed audiograms 1 week before and three months post-surgery. These results were compared and used for statistical analysis using JASP Statistics Software (Sir Harold Jeffreys' Statistics Program, University of Amsterdam). Successful reconstruction was determined by a post-operative air-bone gap of $20 \mathrm{~dB}$ or less $[1,4]$. A Paired Sample T-test with a Wilcoxon signed rank correction was done for statistical validity.

\section{Results and Analysis}

Forty-three patients underwent partial ossicular chain reconstruction during the study period. This cohort comprised both adults and children with ages ranging from 7 to 77 years (Figure 1). Of these 8 were children and 35 were adults. Follow up ranged from 6 months to 3 years with a mean of 22 months. The indications for an Ossicular Chain Reconstruction are summarized in Table 1. The most common indication overall was cholesteatoma followed closely by chronic suppurative otitis media (CSOM) without cholesteatoma.

Table 1: Indication for ossicular chain reconstruction.

\begin{tabular}{|c|c|c|c|}
\hline $\begin{array}{c}\text { Type of } \\
\text { Prosthesis }\end{array}$ & Indication & Frequency & Percent \\
\hline \multirow{4}{*}{ Clip } & Cholesteatoma & 12 & 46.1 \\
\hline & $\begin{array}{l}\text { CSOM without } \\
\text { Cholesteatoma }\end{array}$ & 10 & 38.4 \\
\hline & Traumatic dislocation & 3 & 11.5 \\
\hline & Revision prosthesis & 1 & 3.9 \\
\hline \multirow{4}{*}{ Bell } & Cholesteatoma & 4 & 23.5 \\
\hline & $\begin{array}{l}\text { CSOM without } \\
\text { Cholesteatoma }\end{array}$ & 12 & 70.6 \\
\hline & Traumatic dislocation & 1 & 5.9 \\
\hline & Revision prosthesis & 0 & 0 \\
\hline
\end{tabular}




\section{Overall hearing results}

The preoperative and the postoperative audiogram are included in our statistical analyses. The pure tone audiogram (PTA) and the air-bone gap (ABG) included 0.5, 1, 2 and $3 \mathrm{kHz}$ [1,5-7]. The overall hearing thresholds for the PTA and ABG values are shown in Table 2. The overall PTA improved by $20 \mathrm{~dB}+/-0.63$ (preop: $52.6+/-19$, postop: $32.6+/-14.8$ ), which is statistically significant. The overall ABG improvement was $33.9+/-11.4$ (preop: $13+/-7.6$, postop: $20.7+/-3.8$ ). There was a very small difference in improvement between the results in Clip and Bell group for PTA $(21.6 \mathrm{~dB}+/-4.1$ vs $22.3 \mathrm{~dB}+/-4$ ). A small difference between the results in Clip and Bell groups for ABG (21.8 dB +/- vs $21.2+/-0.4 \mathrm{~dB})$ was also noted. Overall, A successful postoperative ABG of $<20 \mathrm{~dB}$ was obtained in $91 \%$ of the patients $(n=39)$ (Table 3 and Figure 2). The Clip prosthesis had a significantly higher success rate of $96 \%(n=26)$ in comparison to the Bell prosthesis which was successful in $82 \%$ of patients $(n=14)(p<0.001)$. Furthermore, closure of the $A B G$ to $<10 \mathrm{~dB}$ was achieved in $61 \%$ of patients implanted with the Clip prosthesis in contrast to only $36 \%$ of those with the Bell prosthesis. A plot diagram of the paired sample T-Test comparing PTA pre and post-surgery and ABG pre and post-surgery comparing Clip and Bell prostheses show that although both prosthesis succeed in improving PTA and ABG post-operatively, the Air Bone Gap is slightly more reduced using the Clip-Piston prosthesis (Figures 3 \&4). Repeated measures Anova with a Post Hoc test was used in order to compare the two prostheses. The difference between the two prosthesis was statistically significant ( $p<0.01$ ). The Clip prosthesis showed a moderate effect size (Cohen's $d=-0.42$ ) when comparing the PTA results (Table 5) and a small effect size (Cohen's $d=-0.25$ ) in comparison of the ABG (Table 6).

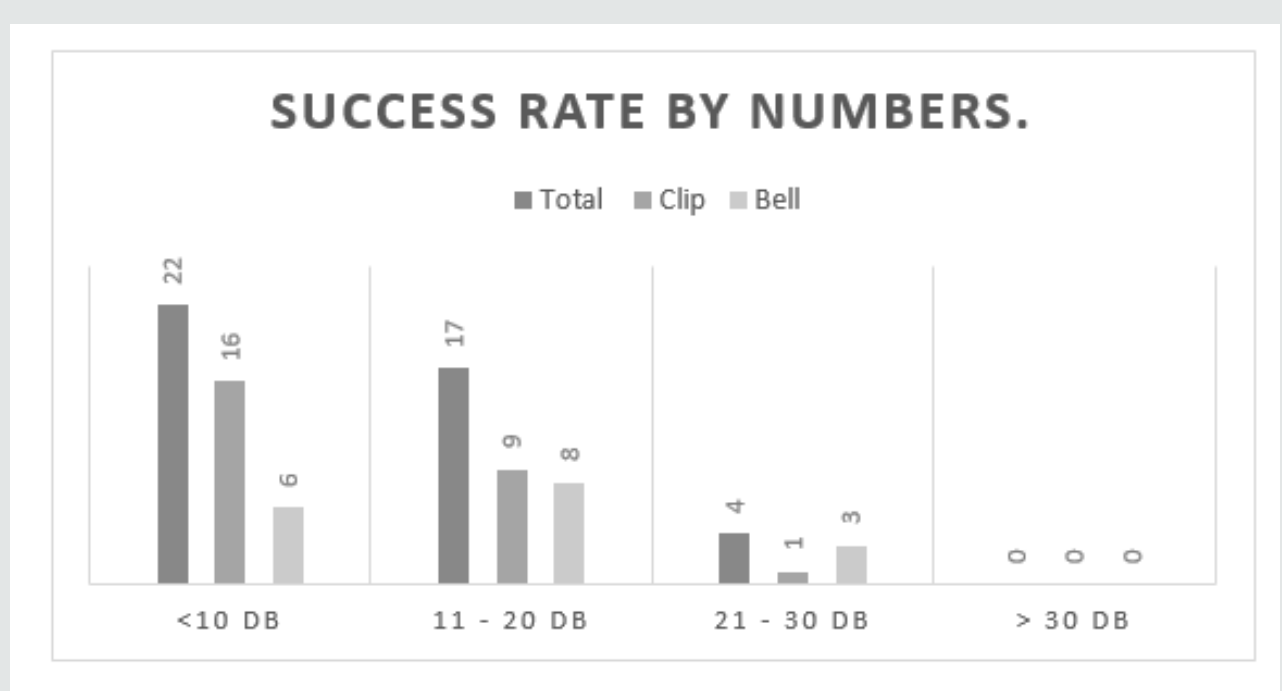

Figure 3: Hearing results by Procedure.

PTA PRE OP - PTA POSTOP
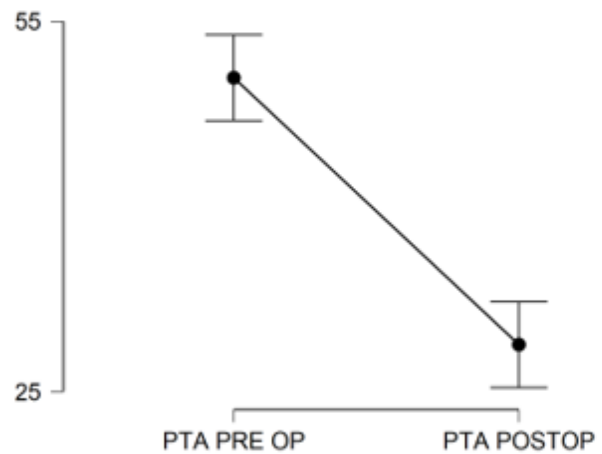

ABG PREOP - ABG POSTOP
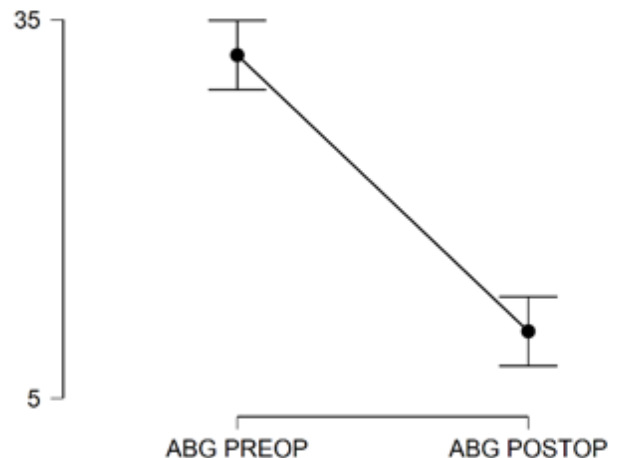

Note that the ' $y$ ' axis unit is Decibels $(d B)$

Figure 4: Plots of PTA pre-surgery and post-surgery and ABG pre- and post-surgery using CLIP. 


\section{PTA PRE OP - PTA POSTOP}
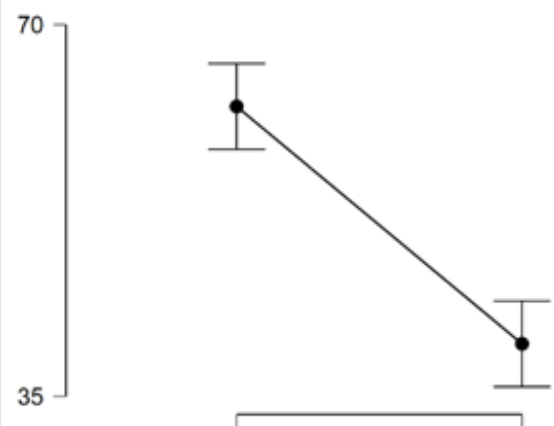

PTA PRE OP

PTA POSTOP

\section{ABG PREOP - ABG POSTOP}
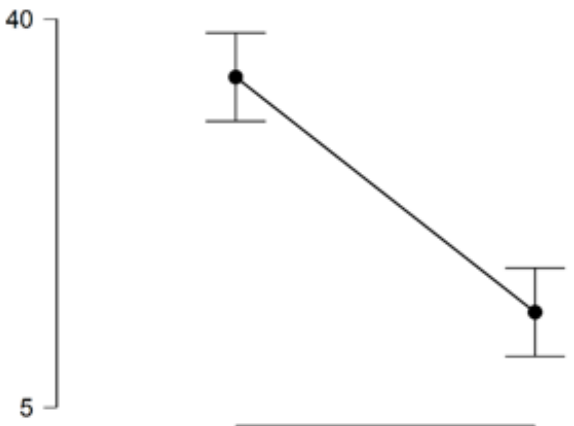

ABG PREOP

ABG POSTOP

Figure 5: Plots of PTA pre- and post-surgery and ABG pre- and post-surgery using BELL.

Table 2: Mean overall hearing results.

\begin{tabular}{|c|c|c|c|}
\hline & Clip (n=26) & Bell $(n=17)$ & Overall $(n=43)$ \\
\hline \multicolumn{4}{|c|}{ PTA $(0.5,1,2,3 \mathrm{kHz})$} \\
\hline Pre & $50.4 \mathrm{~dB}+/-16$ & $62.2 \mathrm{~dB}+/-13$ & $52.6 \mathrm{~dB}+/-19$ \\
\hline Post & $28.8 \mathrm{~dB}+/-11.9$ & $39.9 \mathrm{~dB}+/-17$ & $32.6 \mathrm{~dB}+/-14.8$ \\
\hline \multirow[t]{2}{*}{ Improvement } & $21.6 \mathrm{~dB}+/-4.1$ & $22.3+/-4$ & $20 \mathrm{~dB}+/-4.2$ \\
\hline & $\mathrm{P}<0.01$ & $P<0.01$ & $P<0.001$ \\
\hline \multicolumn{4}{|c|}{ ABG $(0.5,1,2,3 \mathrm{kHz})$} \\
\hline Pre & $32.1 \mathrm{~dB}+/-8.9$ & $34.7 \mathrm{~dB}+/-7.8$ & $33.9 \mathrm{~dB}+/-11.4$ \\
\hline Post & $10.3 \mathrm{~dB}+/-5.9$ & $13.5 \mathrm{~dB}+/-7.4$ & $13.0 \mathrm{~dB}+/-7.6$ \\
\hline \multirow[t]{2}{*}{ Improvement } & $21.8 \mathrm{~dB}+/-3$ & $21.2 \mathrm{~dB}+/-0.4$ & $20.7 \mathrm{~dB}+/-3.8$ \\
\hline & $P<0.001$ & $P<0.001$ & $P<0.001$ \\
\hline
\end{tabular}

Table 3: Success rates: Defined in ABG $<20 \mathrm{~dB}$ according to AA0-HNS Guidelines.

\begin{tabular}{|c|c|c|c|}
\hline & Total $(n=43)(\%)$ & Clip (n=26) (\%) & Bell $(n=17)(\%)$ \\
\hline \multicolumn{4}{|c|}{ ABG $(0.5,1,2,3 \mathrm{kHz})$} \\
\hline$<10 \mathrm{~dB}$ & $51 \%(n=22)$ & $61 \%(n=16)$ & $36 \%(n=6)$ \\
\hline$<20 \mathrm{~dB}$ & $40 \%(\mathrm{n}=17)$ & $35 \%(n=9)$ & $47 \%(n=8)$ \\
\hline$<30 \mathrm{~dB}$ & $9 \%(n=4)$ & $4 \%(n=1)$ & $17 \%(n=3)$ \\
\hline
\end{tabular}

Table 4: Post Hoc Comparisons - Type of Prosthesis PTA.

\begin{tabular}{|c|c|c|c|c|c|c|c|c|}
\hline \multicolumn{9}{|c|}{ 95\% CI for Mean Difference } \\
\hline & & $\begin{array}{c}\text { Mean } \\
\text { Difference }\end{array}$ & Lower & Upper & SE & $\mathbf{t}$ & Cohen's d & p holm \\
\hline Clip & Bell & -11.502 & -19.96 & -3.044 & 4.188 & -2.746 & -0.419 & $0.009 * *$ \\
\hline
\end{tabular}

Table 5: Post Hoc Comparisons - Type of Prosthesis ABG.

\begin{tabular}{|c|c|c|c|c|c|c|c|c|}
\hline \multicolumn{9}{|c|}{ 95\% CI for Mean Difference } \\
\hline & & $\begin{array}{c}\text { Mean } \\
\text { Difference }\end{array}$ & Lower & Upper & SE & $\mathbf{t}$ & Cohen's d & p holm \\
\hline Clip & Bell & --2.926 & -6.478 & 0.625 & 1.759 & -1.664 & -0.254 & 0.104 \\
\hline
\end{tabular}




\section{Complications}

One patient in the Clip prosthesis cohort required a revision procedure due to the inadequate length of the prosthesis. This was replaced by a longer prosthesis without complication. No patients experienced extrusion of their prostheses or sensorineural hearing loss. One revision OCR in the Bell cohort was needed as it extruded, subsequently it was replaced with a Clip prosthesis. No patients experienced sensorineural hearing loss.

\section{Discussion}

The titanium Clip has been designed to improve operative and hearing outcomes over previous generation of prostheses developed for ossicular chain reconstruction [8]. With flexible feet at the base of the prosthesis, a secure coupling can be achieved with the stapes capitulum with ease of application leading to reduced displacement and improved sound conduction [9-11]. This study aimed to evaluate the audiological outcomes and safety profile of this prosthesis and compare this to the earlier Bell prosthesis.

Our overall results demonstrate a significant improvement in hearing outcomes with both prostheses. The average improvements in the ABG for the Clip and Bell prosthesis were $21.8 \mathrm{~dB}$ and $21.2 \mathrm{~dB}$, respectively. Similarly, favorable outcomes have also been reported in the literature. In their study of 130 patients receiving the Clip prosthesis, Kahue et al. [12] observed a reduction in the median $\mathrm{ABG}$ and PTA of $11 \mathrm{~dB}$ in comparison to preoperative values. Similarly, Gostian et al. [13] demonstrated stable hearing outcomes in their long-term study (6.5 years) where a reduction in ABG of $8.9 \mathrm{~dB}$ was seen. In our cohort of patients receiving using the Clip prosthesis, successful ossicular reconstruction, defined as an ABG of $20 \mathrm{~dB}$ or less, was achieved in $91 \%$ of patients. This was a significant improvement over the Bell prosthesis which exhibited a success rate of $82 \%$. Furthermore, an $\mathrm{ABG}$ of $10 \mathrm{~dB}$ or less was achieved in $61 \%$ of patients in the Clip versus just $36 \%$ of those in the Bell cohort. The superiority of these outcomes is substantiated by the previously mentioned authors where success rates of $72 \%$ [13] and 63\% [12] were achieved using the Clips prosthesis.

We that found that one patient experienced extrusion of the Bell prosthesis despite the use of cartilage interposition grafts. No such cases were experienced with the Clip prosthesis however one revision was required with this device due to inadequate length and persistent hearing loss. The Clip prosthesis was able to be replaced without any undue force on the stapes or subsequent complications. These low rates of displacement/extrusion compare far more favorable that those reported with earlier generation prostheses which have demonstrated displacement in up to $7.7 \%$ of cases [14]. This is likely the consequence of the clip design optimizing adherence to the stapes superstructure. The design also requires a small amount of force to engage the clip onto the stapes capitulum. In our study, this did not result in disruption of the stapes or sensorineural hearing loss in any of our patients which supports the safety profile of its application. Indeed, in the two compared studies no significant events were reported as a result of the surgery and a minor extrusion rate of $1.5 \%$ in patients which was seen in the setting of recurrent middle ear disease [12]. Several limitations can be described in this study. The retrospective nature of this investigation lends itself to inherent selection bias. This in addition to the small cohort size may influence the significance of the statistical analysis. With further recruitment and longer term follow up more definitive conclusions can be drawn and provide opportunity for subgroup analysis.

\section{Conclusion}

Partial ossicular chain reconstruction using the titanium Clip prosthesis provides excellent audiological outcomes superior to those seen with the earlier generation Bell prosthesis. Successful hearing restoration was seen in $91 \%$ of patients with a favorable safety profile. No cases of displacement, extrusion or sensorineural hearing loss were encountered.

\section{References}

1. Monsell MBTA, Gates GA, Goldenberg RA, Meyerhoff WL, House JW (1995) Committee on Hearing and Equilibrium guidelines for the evaluation of results of treatment of conductive hearing loss. Otolaryngol Head Neck Surg 113(3): 186-187.

2. Statistics ABO (1993) Disability, Ageing and Cares. Hearing Impairment.

3. Hogan A, Shipley M, Strazdins L (2001) Communication and behavioral disorders among children with hearing loss increased risk of mental health disorders. . Australian \& NZ Journal of Public Health 35(4): 377383.

4. Shah KD, Bradoo RA, Joshi AA, Sapkale DD (2013) The efficiency of titanium middle ear prosthesis in ossicular chain reconstruction: our experience. Indian J Otolaryngol Head Neck Surg 65(4): 298-301.

5. Haberman RS, Salapatas AM (2018) Hearing Outcomes after Ossicular Reconstruction with Removal of the Malleus. Otolaryngol Head Neck Surg 158(1): 144-150.

6. Roth JA, Pandit SR, Soma M, Kertesz TR (2009) Ossicular chain reconstruction with a titanium prosthesis. J Laryngol Otol 123(10): 1082-1086.

7. Vassbotn FS, Moller P, Silvola J (2007) Short-term results using Kurz titanium ossicular implants. Eur Arch Otorhinolaryngol 264(1): 21-25.

8. Gottlieb PK, Li X, Monfared A, Blevins N, Puria S (2016) First results of a novel adjustable length ossicular reconstruction prosthesis in temporal bones. Laryngoscope 126(11): 2559-2564.

9. Mao M, Zhai J, Chen G, Zhang J, Ma Z, Xue J (2014) Effect of ossicular chain reconstruction with titanium ossicular replacement prosthesis in mastoidectomy with synchronous ossiculoplasty. Lin Chung Er Bi Yan Hou Tou Jing Wai Ke Za Zhi 28(10): 708-711.

10. Goode RL, Nishihara S (1994) Experimental models of ossiculoplasty. Otolaryngol Clin North Am 27(4): 663-675.

11. Birk S, Brase C, Hornung J (2014) Experience with the use of a partial ossicular replacement prosthesis with a ball-and-socket joint between the plate and the shaft. Otol Neurotol 35(7): 1248-1250. 
12. Kahue CN, O Connnell BP, Dedmon MM, Haynes DS, Rivas A (2018) Short and Long-Term Outcomes of Titanium Clip Ossiculoplasty. Otol Neurotol 39(6): e453-e460.

13. Gostian AO, Kouame JM, Bremke M, Ortmann M, Huttenbrink KB, et al. (2016) Long term results of the titanium clip prosthesis. Eur Arch Otorhinolaryngol 273(12): 4257-4266.

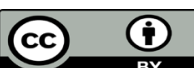

This work is licensed under Creative Commons Attribution 4.0 License

To Submit Your Article Click Here: Submit Article
14. Hillman TA, Shelton C (2003) Ossicular chain reconstruction: titanium versus plastipore. Laryngoscope 113(10): 1731-1735.

DOI: $10.32474 /$ SJ0.2020.05.000207

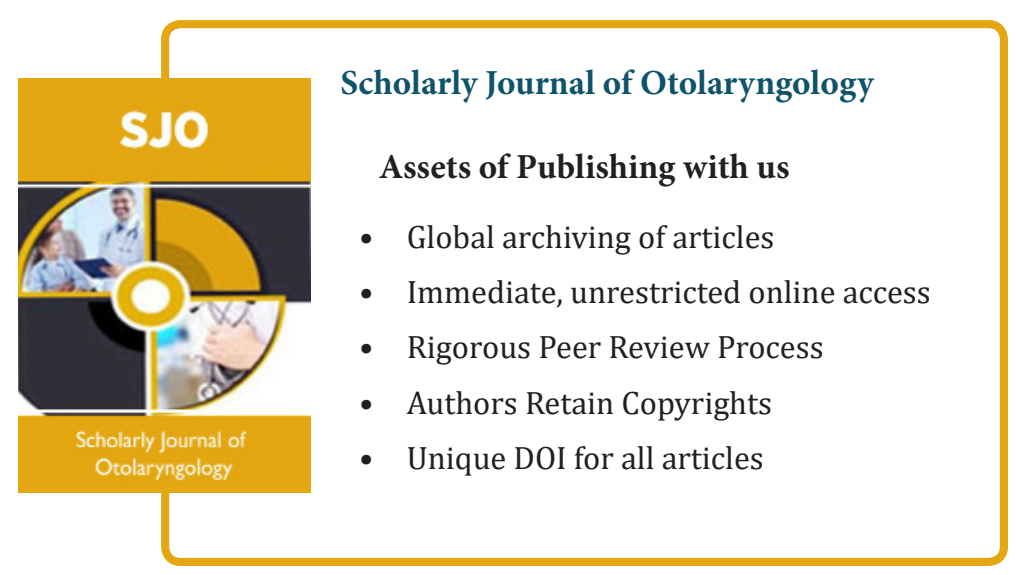

